\title{
A NEW PATCH-BASED APPROACH FOR NOISE FILETRING
}

\author{
Quoc Bao DO, Azeddine BEGHDADI, Marie LUONG \\ L2TI, Institut Galilée, Université Paris 13, France
}

\begin{abstract}
A common framework is proposed for Gaussian, unilateral, bilateral and non-local means filters. A new denoising method is then introduced for removing additive independent Gaussian noise. It is adapted and applied for filtering signal-dependent noise such as film grain noise. The main idea is to transform the signal-dependent into independent additive Gaussian noise using a nonlinear transformation prior to filtering. The obtained results clearly demonstrate the efficiency of the proposed method.
\end{abstract}

Index Terms - Denoising, film degrain, nonlocal processing, image restoration, photographic film.

\section{INTRODUCTION}

Photographic films usually suffer from a particular noise, namely "grain noise", due to silver halide crystals that clump together in the development process. This noise or graininess is much visible in uniform areas and may also affect some salient features of the signal. In the classical model, the noise resulting from scanning a photographic film is usually assumed to be an additive, zero mean, signaldependent Gaussian noise [1]. A non linear transformation [2] is then used in order to obtain an independent additive noise model, before applying a classical denoising method. For denoising such independent noise, several filtering methods have been proposed in the literature. Here, we investigate some filters such as the Gaussian[1] (GAU), unilateral[3] (UNI), bilateral[4] (BIL) and non-local means [5] (NLM) filters. The GAU filter uses only spatial information whereas the BIL and UNI filters are data dependent. The UNI filter incorporates the pixel intensity similarity in the design of the kernel. The BIL filter uses a kernel where spatial and intensity informations are taken into account. However, these filters are based on a pixel-wise similarity measure and consequently do not take into account the contextual and spatial information. To cope with this limitation, NLM filter uses block-based similarity approach. Other interesting methods, such as total variation approaches and wavelet thresholding have been proposed as alternative [6,7]. Here we focus on non-local approaches such as the methods proposed in [1],[3]-[5]. We will demonstrate that they could be expressed in a common framework. A comparative study is then performed and a new denoising filter is proposed for removing both independent and dependent noises.

The paper is organized as follows. A review and an unified framework are presented in section 2. The proposed algorithm for removing independent noise is described in section 3. In section 4, our proposed film degrain method is introduced. The experimental results are reported in section 5.

\section{A COMMON FRAMEWORK}

First, let us define some notations: $I_{p}, I_{q}$ are intensities of pixel $p$ and $q$ respectively; $f_{p}, f_{q}$ are two small patches defined for pixel $p$ and $q$ respectively; $\|a\|_{2}^{2}$ is the Euclidean norm of $a$; For GAU filter, the estimated value of pixel $p$ is defined as follows:

$$
I_{p}^{G A U}=\frac{\sum_{q} G\left(\|p-q\|_{2}^{2}, \sigma_{s}^{2}\right) I_{q}}{\sum_{q} G\left(\|p-q\|_{2}^{2}, \sigma_{s}^{2}\right)}
$$

where, $G\left(u, \sigma^{2}\right)=\exp \left(-u / \sigma^{2}\right)$ and $\sigma_{s}$ is the spatial standard deviation. This filter uses the Gaussian weighted average of its neighbors (called spatial neighbors). Instead of using coordinates $p, q$, UNI filter proposes to use intensity $I_{p}, I_{q}$ :

$$
I_{p}^{U N I}=\frac{\sum_{q} G\left(\left\|I_{p}-I_{q}\right\|_{2}^{2}, \sigma_{r}^{2}\right) I_{q}}{\sum_{q} G\left(\left\|I_{p}-I_{q}\right\|_{2}^{2}, \sigma_{r}^{2}\right)}
$$

where $\sigma_{r}$ is the range standard deviation. This method alters one pixel by the weighted average of all pixels (called range neighbors) whose intensity is similar to its. BIL filter, it simply combines the above filters as follows:

$$
I_{p}^{B I L}=\frac{\sum_{q} G\left(\|p-q\|_{2}^{2}, \sigma_{s}^{2}\right) G\left(\left\|I_{p}-I_{q}\right\|_{2}^{2}, \sigma_{r}^{2}\right) I_{q}}{\sum_{q} G\left(\|p-q\|_{2}^{2}, \sigma_{s}^{2}\right) G\left(\left\|I_{p}-I_{q}\right\|_{2}^{2}, \sigma_{r}^{2}\right)}
$$

It is well known that GAU filter can eliminate noise but oversmoothes contours and details. Unlike GAU, UNI filter takes into account the range neighbors making thus the filter more robust. BIL algorithm considers both spatial and range neighbors to better preserve details. Note that UNI and BIL 
filters compare intensity of only two pixels using a local similarity measure in order to determine range neighbors. This makes the filters less robust in textured and contrasted regions. To overcome these limitations, NLM filter uses a block-based similarity measure for estimating the output value as follows:

$$
I_{p}^{N L M}=\frac{\sum_{q} G\left(\left\|f_{p}-f_{q}\right\|_{2}^{2}, \sigma_{r}^{2}\right) I_{q}}{\sum_{q} G\left(\left\|f_{p}-f_{q}\right\|_{2}^{2}, \sigma_{r}^{2}\right)}
$$

This equation is similar to (2) except that $f_{p}$ and $f_{q}$ are used instead of $I_{p}$ and $I_{q}$. It has been reported that blockbased methods are more robust than pixel-based methods [8]. Indeed, by exploiting neighborhood information in the filter design, a good discrimination between noise and signal is achieved. It is worth to notice that the cited filters share some properties. We propose here, to reformulate these filters in a common framework as follows:

$$
I_{p}^{U I F}=\frac{\sum_{q} G\left(\|p-q\|_{2}^{2}, \sigma_{s}^{2}\right) G\left(\left\|v f_{p}-v f_{q}\right\|_{2}^{2}, \sigma_{r}^{2}\right) I_{q}}{\sum_{q} G\left(\|p-q\|_{2}^{2}, \sigma_{s}^{2}\right) G\left(\left\|v f_{p}-v f_{q}\right\|_{2}^{2}, \sigma_{r}^{2}\right)}
$$

We introduce a new parameter $v$ which is simply a small block of the same size as $f_{p}$ and $f_{q}$. For pixel-based similarity approach, $v$ equals to 1 at the center and 0 otherwise. For block-based similarity methods, $v$ is set to 1 everywhere. In the case of spatial neighbors filters, $\sigma_{r}$ is set to $+\infty$, and in the case of range neighbors algorithms, $\sigma_{s}$ is equal to $+\infty$. This is summarized in table 1 and table 2.

\begin{tabular}{|c|c|c|c|c|c|}
\hline Method & GAU & UNI & BIL & NLM & PUF \\
\hline Spatial Neighbors & Yes & & Yes & & Yes \\
\hline Range Neighbors & & Yes & Yes & Yes & Yes \\
\hline Pixel-based similarity & & Yes & Yes & & \\
\hline Block-based similarity & & & & YES & Yes \\
\hline
\end{tabular}

Table 1: Brief description of filter types

\begin{tabular}{|c|c|c|c|}
\hline Method & $\sigma_{s}$ & $\sigma_{r}$ & $v$ \\
\hline GAU & & $+\infty$ & \\
\hline UNI & $+\infty$ & & 1 at the center, 0 otherwise \\
\hline BIL & & & 1 at the center, 0 otherwise \\
\hline NLM & $+\infty$ & & 1 everywhere \\
\hline
\end{tabular}

Table 2: Filters and relevant parameters.

\section{PROPOSED DENOISING METHOD}

Here, we propose a novel denoising method for additive, independent Gaussian noise. As can be seen in table 1, NLM filter does not take into account the spatial neighborhood factor which effectively plays an important role in BIL filter. Indeed, among two pixel-based similarity approaches, i.e. BIL and UNI filters, the former outperforms the latter (see the experience section). This is a clue to show crucial role of the spatial neighborhood factor. Despite the fact that NLM filter has been extended from pixel-based similarity approach to block-based similarity one to determine range neighbors, the Euclidean norm is not effective enough to estimate the block similarity [6,7]. For instance, in Fig.1, the intensity Euclidean distance of four blocks b1, b2, b3, b4 (blue ones) to block b0 (yellow one) is computed. Even these four blocks have different structures, they have all similar Euclidean distance to block b0. Consequently, NLM method yields the same weight for these four blocks, resulting thus to over smoothing effect. Note that among these (blue) blocks, only b1 has similar structure as b0 thanks to the continuity property of image (i.e. two blocks closer in space are more likely to contain similar structures). Therefore, b1 should have greater weight than b2, b3 and b4. To this end, we need to integrate the spatial neighborhood factor into equation (4). Finally, our proposed filter called PUF (Proposed Unified Filter) is as follows:

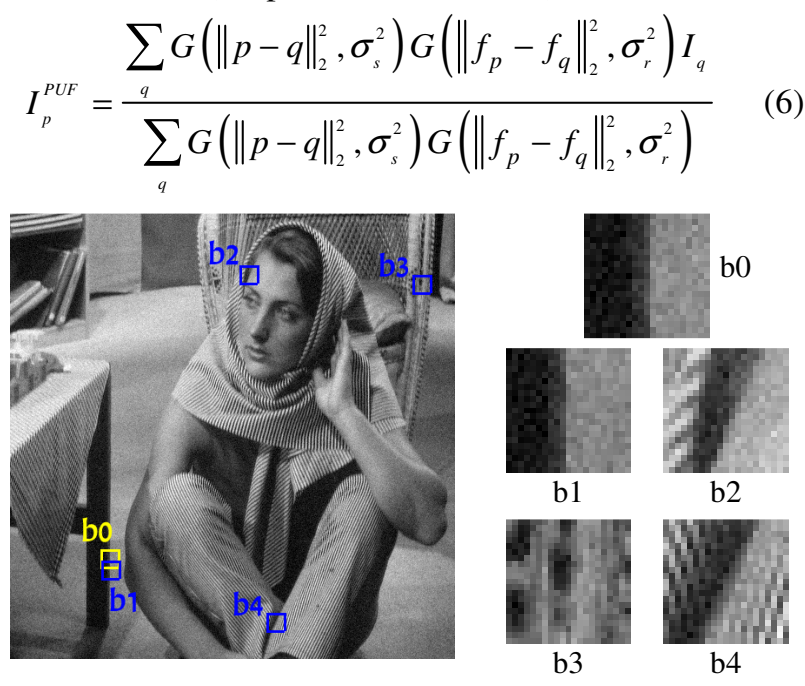

Fig. 1: The ineffectiveness of Euclidean distance: b1, b2, b3, b4 are four different blocks but have similar Euclidean distance to b0 (959.31, 958.98, 958.93 and 959.14 respectively)

\section{FILM IMAGE DEGRAINING}

In the classical model, the grain noise in the digitalized film is usually considered as an additive, zero mean, signaldependent Gaussian noise[1]:

$$
u=s+n
$$

where $s$ is the signal to estimate, $u$ is the observed signal, $n$ is a Gaussian noise with zero mean and standard deviation $k \sqrt{s}$ ( $k$ is scalar constant dependent on the ratio of the grain size to the scanning aperture). Denoising such a signaldependent noise is difficult task. Some approaches have been then proposed to recast the original problem into an independent noise model [9]. In [2], an original solution has been proposed. The input signal is transformed using a non linear transform as follows:

$$
u_{t}=h \sqrt{u}
$$


where $h$ is constant. Under practical conditions, the scanning aperture is much larger than the grain size, resulting in very small value $k$. In this context, by using the transformation (8), the model in equation (7) becomes:

$$
u_{t}=s_{t}+n_{t}
$$

where $s_{t}$ is the new signal to estimate, $u_{t}$ is the new observed signal, $n_{t}$ is a Gaussian noise with zero mean and standard deviation $h k / 2$. Obviously, the noise $n_{t}$ is no longer dependent on the signal $s_{t}$. To evaluate the performance of the transformation, a digital image (shown in Fig.2) obtained from scanning a photographic one is tested.

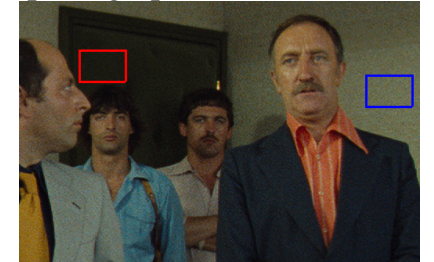

Fig.2: A scanned photographic image

To estimate grain noise characteristics, we select two homogenous regions where one (blue rectangle) is brighter than the other (red rectangle). The intensity distributions of the two regions are shown in Fig.3 (left). It is observed that the intensity distribution in the two regions is Gaussian but with different standard deviation. In the darker region (red profile), the standard deviation is 3.24 whereas in the brighter one (blue profile), this value is greater, i.e. 7.11. It means that the noise is signal-dependent.

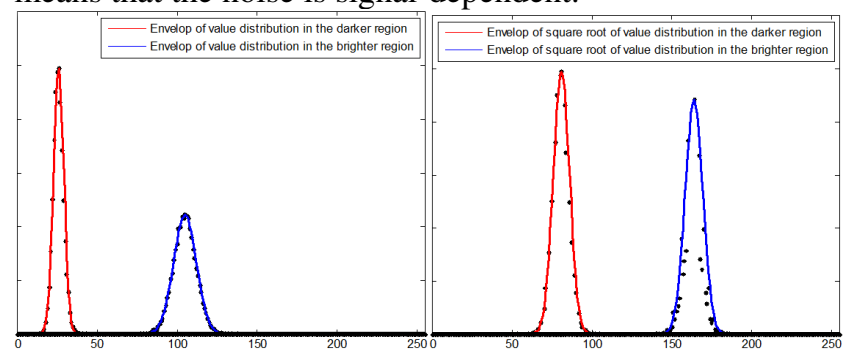

Fig.3: (left) Intensity distribution of the selected regions defined in Fig.2; (right) The corresponding distributions of the transformed intensity

By using the nonlinear transformation in equation (8) (with $h=16$ ), the two intensity distributions have the same shape as shown in Fig.3(right). As can be seen, they have Gaussian distribution with the similar standard deviation (around 5.50). This means that the new noise is no longer signal-dependent. Therefore, instead of estimating directly $u$ from equation (7), we will tackle equation (9) to find $u_{t}$ by using the proposed method in section 3. Once $u_{t}$ has been estimated, $u$ can be easily computed.

\section{EXPERIMENTAL RESULTS}

To evaluate the performance of our method, some experiments are carried out on simulated images perturbed by additive, independent Gaussian noise as well as on real images affected by grain noise. In the first case, only three UNI, BIL, NLM filters are used for comparison. Lena,
Barbara, Pepper images are corrupted by additive, independent Gaussian noise of standard deviation $\sigma_{n}=10$. The parameter $\sigma_{s}$ is set to 3.87 for all filters. The range parameter $\sigma_{r}$ in the pixel-based similarity methods, i.e. UNI and BIL, is set to 30. For block-based similarity methods, i.e. NLM and PUF, $\sigma_{r}$ is set to 10 . We compare, both objectively and subjectively, the performance of the methods. For objective evaluation, two fidelity metrics MAD [10] and $\operatorname{PSNR}_{\mathrm{W}}$ [11] which are based on the visual human system are used. Note that the smaller the value of MAD, the better the image quality. However, the higher the value of PSNR $\mathrm{W}$, the better the image quality. As reported in table 3, the proposed method outperforms the others. Indeed, in many cases, our method is the best, NLM is the $2^{\text {nd }}$, BIL is $3^{\text {rd }}$ and UNI is the worst. As can be seen in Fig.4, for UNI yields oversmoothing effect. Whereas, BIL is better than UNI but sand-like artifacts still remain. These artifacts do not appear in NLM method but an oversmoothing results in nearly homogeneous regions (see circles in Fig.4e). The proposed filter yields the best result since noise is effectively eliminated and details are well preserved. Fig.5 presents the differences between the noisy image and the restored ones. For an efficient filtering this image must contain only noise component. As can be seen, the difference images corresponding to UNI, BIL, NLM methods still contain some signal structures. Whereas, the one corresponding to the proposed method contains essentially noise structures.

\begin{tabular}{|c|c|c|c|c|c|c|}
\hline & \multicolumn{3}{|c|}{ MAD } & \multicolumn{3}{c|}{ PSNR $_{\mathrm{W}}$} \\
\cline { 2 - 7 } & Lena & Barbara & Pepper & Lena & Barbara & Pepper \\
\hline UNI & 5.14 & 5.12 & 5.71 & 13.29 & 12.79 & 13.90 \\
\hline BIL & 2.33 & 2.47 & 2.75 & 15.71 & 14.65 & 16.82 \\
\hline NLM & 2.13 & 2.17 & 3.23 & 16.35 & 16.26 & 16.85 \\
\hline PUF & $\mathbf{1 . 6 3}$ & $\mathbf{1 . 7 1}$ & $\mathbf{2 . 6 7}$ & $\mathbf{1 6 . 9 4}$ & $\mathbf{1 6 . 4 1}$ & $\mathbf{1 7 . 4 7}$ \\
\hline
\end{tabular}

Table 3: Objective measures (bold letters mean the best result)

The NLM, PUF methods and a commercial software, namely NeatImage [12] are considered in the second test with real images affected by grain noise. Note that in this case, the nonlinear transformation (see equation (8)) is firstly carried out to obtain an independent Gaussian noise model. Three restored images as well as three differences between the square root of the original image and the square root of the restored images are given in Fig.7b,c,d and Fig.7e,f,g respectively. From these figures, we can notice that the proposed filter outperforms the others. Indeed, the image difference signal is close to noise while the others contain some signal details. (More results are also available on http://www-l2ti.univ-paris13.fr/ do/WoSSPA/index.html)

\section{REFERENCES}

[1] K.R. Castelman, Digital image processing, Prentice Hall International Editions, Englewood Cliffs, New Jersey, 1996.

[2] H.H. Arsenault, C. Gendron, M. Denis, "Transformation of film grain noise into signal independent additive Gaussian noise", Journal of the optical Society of America, pp. 91-94, 1981. 
[3] L.P. Yaroslavsky, "Digital Picture Processing - An Introduction", Springer Verlag, 1985.

[4] C. Tomasi, R. Manduchi, "Bilateral Filtering for Gray and Color Images" in Proc. ICCV, pp.839, 1998.

[5] A. Buades, B. Coll, and J. Morel, "A Non-Local Algorithm for Image Denoising", in Proc. CVPR, pp.60-65, 2005.

[6] J.-L. Starck, E. J. Candès, and D. L. Donoho, "The Curvelet Transform for Image Denoising," IEEE Transactions on Image Processing, vol. 11, no. 6, pp. 670-684, Jun. 2002.

[7] L.I. Rudin, S. Osher, E. Fatemi, "Nonlinear total variation based noise removal algorithms", Physica D, vol. 60, pp. 259-268, 1992.
[8] V. Katkovnik, A. Foi, K. Egiazarian, and J. Astola, "From local kernel to nonlocal multiple-model image denoising", Int. J. Computer Vision, vol. 86, no. 1, pp. 1-32, 2010.

[9] R. Kasturi, J.F. Walkup, and T.F. Krile, "Image restoration by transformation of signal-dependent noise to signal-independent noise," Appl. Opt. 22, pp. 3537-3542, 1983.

[10] E.C. Larson, D.M. Chandler, "Most apparent distortion: fullreference image quality assessment and the role of strategy," Journal of Electronic Imaging, 19 (1), 2010.

[11] A. Beghdadi, B. Pesquet-Popescu, "A New Image Distortion Measure Based Wavelet Decomposition," In Proc. ISSPA, pp.485488, 2003.

[12] http://www.neatimage.com

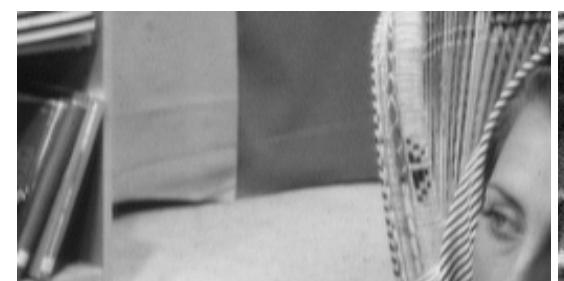

(a) The original image

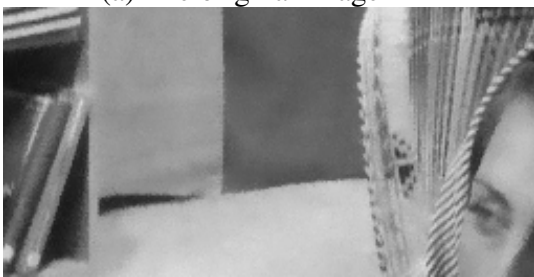

(d) BIL's result

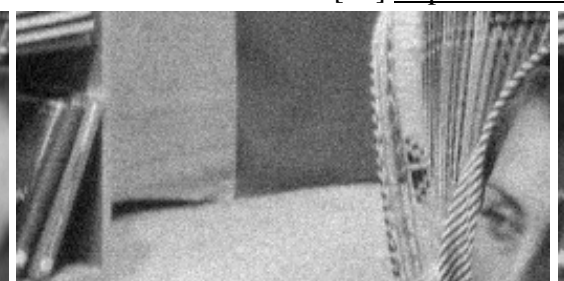

(b)The noisy image

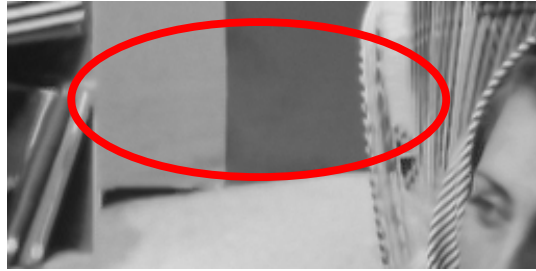

(e) NLM's result

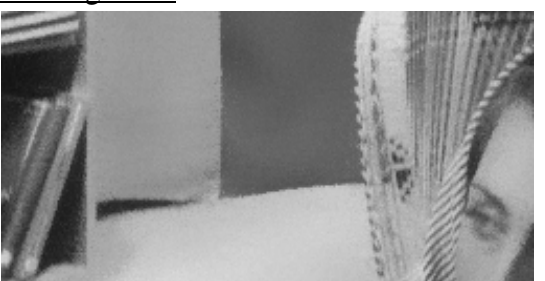

(c) UNI's result

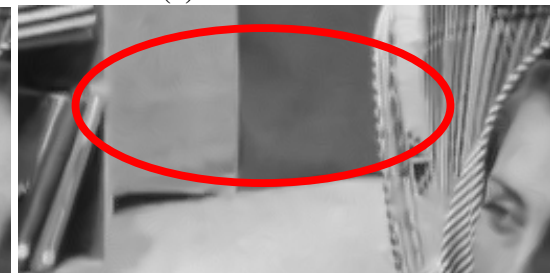

(f) PUF's result

Fig. 4: Results for Barbara image

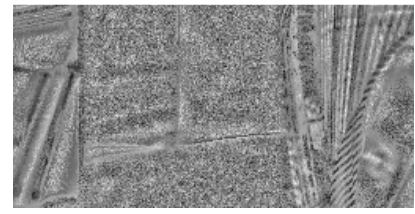

(a) Difference of UNI/noisy image

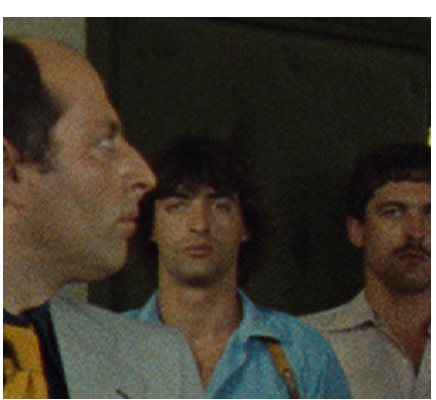

(a) The original image

Fig. 6: Results of degrain noise. (e), (f), (g) are differences between the square root of the original image and the square root of the three restored ones, respectively

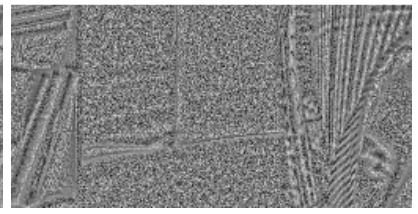

(b) Difference of BIL/noisy image Fig.5: Differences between the noisy image and the restored ones

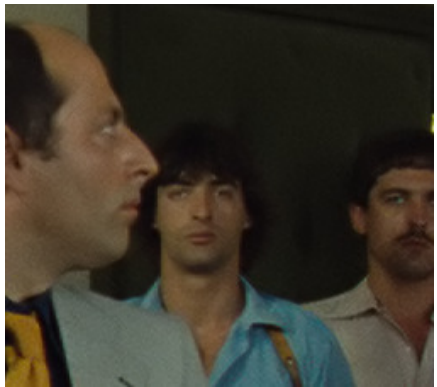

(b) NeatImage's result

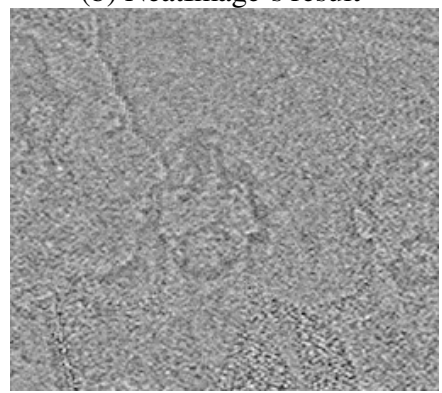

(e)

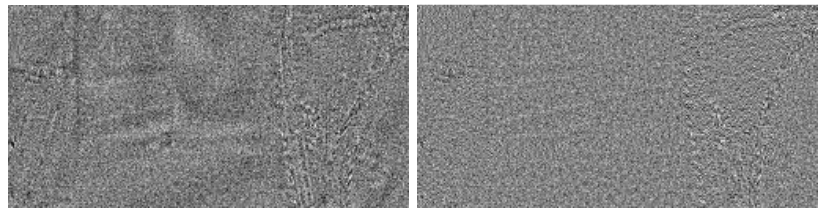

c) Difference of NLM/noisy image (d) Difference of PUF/noisy image

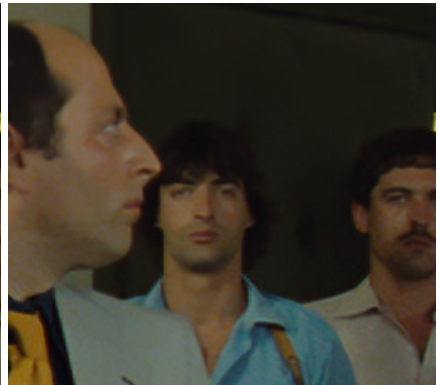

(c) NLM's result

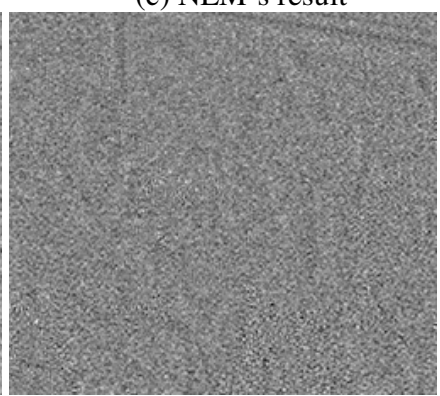

(f)

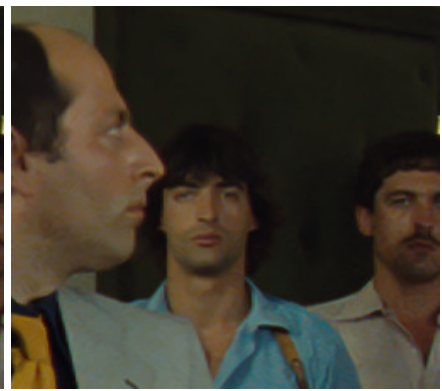

(d) PUF's result

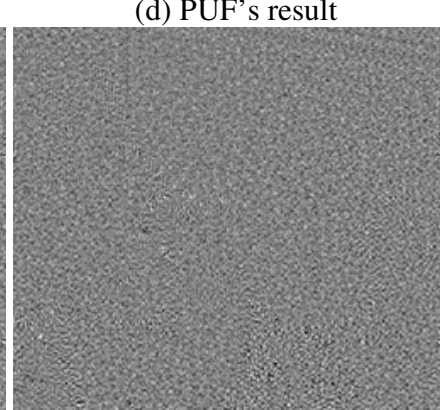

(g) 